\title{
Las nuevas necesidades de la comunicación política en México
}

\section{Elías Aguilar García ${ }^{1}$}

El resultado de las elecciones presidenciales del 2 de julio del año 2000 en México significó el golpe de gracia al envejecido y monolítico sistema unipartidista y el arribo definitivo de la alternancia de partidos políticos en los diferentes puestos de elección popular, en especial en el poder Ejecutivo. El actual panorama muestra elecciones reñidas, que se definen en el último trecho de la contienda con márgenes porcentuales mínimos, donde los medios de comunicación -especialmente radio y televisión-conforman cada día más la agenda política en la que los ciudadanos basan su decisión. Éstos son algunos de los rasgos que han adquirido las contiendas electorales en el México contemporáneo.

La mayor equidad entre los partidos en la competencia electoral ha tenido, asimismo, un fuerte impacto en la forma en que los gobiernos de los diferentes partidos políticos se presentan ante los ciudadanos. Con la convicción de que gobernar es comunicar, los diferentes gobiernos en México están invirtiendo elevadas cantidades de recursos en la comunicación, con el fin inmediato de ganarse la aprobación de la ciudadanía.

Es común ver en México campañas creativas e innovadoras que publicitan las obras y logros de los diferentes gobiernos en sus distintos niveles (federal, estatal y municipal). Gobernantes del Partido Acción Nacional (PAN), del Partido Revolucionario Institucional (PRI) y del Partido de la Revolución Democrática (PRD) se han volcado hacia los consultores en mercadotecnia política, con el fin último de allegarse la lealtad de los ciudadanos y persuadirlos de sus sanas intenciones democráticas.

Sin embargo, los resultados no siempre han generado una valoración positiva entre los electores y muchas veces sólo llevan a un gasto inútil de

1. Universidad de las Américas-Puebla, correo electrónico: indicadores_aguilar@hotmail.com. 
recursos, tomando en consideración que no garantizan en absoluto la permanencia de un partido político en puestos de elección popular. Con esto no quiero decir que un sistema funcione con la permanencia de un partido en el poder, pero tampoco que el cambio de partido en cada elección sea la situación ideal. Una democracia de partidos asume que si un partido desarrolla bien su trabajo en el poder, los ciudadanos lo valorarán y entonces le permitirán mantenerse por uno o más periodos, premiando en las urnas su desempeño como gobierno.

En este contexto de cambio y de nuevos escenarios políticos y comunicacionales que permean al México contemporáneo, el presente trabajo tiene como objetivo contribuir al entendimiento de los factores que intervienen en la evaluación que la ciudadanía hace de sus gobernantes. Específicamente se busca responder a algunas interrogantes. En primer lugar, ¿cómo evalúan los ciudadanos mexicanos a sus gobiernos?; ${ }^{2}$ en segundo término, ise perciben diferencias entre gobiernos de diferente filiación partidista?; y como tercer punto, dados los factores analizados, ise puede pensar en un concepto de comunicación que sea algo más que promocionar la obra de gobierno, tan puesto en práctica en los actuales gobiernos municipales y estatales en el país?

El argumento central del trabajo es que, dadas las características de desigualdad en la distribución del ingreso-consumo de México; y las consecuencias en términos de cultura política entre los ciudadanos menos favorecidos del país, un modelo de comunicación centrado exclusivamente en una visión mercadotécnica de la comunicación no es suficiente para que el ciudadano valore las aportaciones de un gobierno. Se requiere de esquemas de desarrollo comunitario que promuevan la resolución de problemas concretos con esquemas de participación democrática.

El trabajo está organizado en seis segmentos. En primer lugar, se dibuja un panorama del sistema político mexicano en las últimas dos décadas -incluidos los cambios que ha experimentado- y se analizan algunos trabajos previos sobre la cultura política del mexicano. En segundo lugar se discute el contexto socioeconómico del ciudadano en el país. En tercer término, se abordan los conceptos "comunicación del sector público" y "desarrollo comunitario". El cuarto punto es una descripción metodológica del trabajo de campo. Posteriormente se presentan algunos resultados relevantes que responden a nuestras preguntas, para finalmente concluir.

2. A pesar de que en los artículos 103 y 104 de la Constitución se especifica que los ayuntamientos están obligados a proporcionar los servicios de: a) agua potable y alcantarillado; b) alumbrado público; c) limpia; d) mercados; e) panteones; f) rastros; g) calles, parques y jardines; $y, h$ ) seguridad pública y tránsito; los ciudadanos les asignan otras funciones. 
Es importante señalar que el presente trabajo no es de carácter conclusivo; más bien plantea más preguntas de las que se responden. Presenta resultados preliminares, cuyo análisis exhaustivo aún se está realizando, dependiendo éste de recursos externos y propios del investigador para desarrollarlo a plenitud.

\section{Los cambios en México}

Los cambios económicos y políticos acaecidos en México durante las últimas tres décadas han sido significativos, y explican en gran medida la situación por la que transitamos en la actualidad. Sin pretender abarcar a detalle los cambios, y con el fin de ubicar en un contexto la situación actual en que está el sistema político mexicano, a continuación se hace un breve recuento de la transformación de México.

\section{Orientación hacia el mercado externo}

El proceso de transformación se inició en el decenio de 1960, cuando el modelo denominado desarrollo estabilizador dio las primeras señales de desgaste y obsolescencia; simbólicamente, diversos autores lo ubican en el movimiento estudiantil de 1968 (Grayson, 2001; Shatz, 2000; Stevens, 1974).

El modelo de sustitución de importaciones basado en un esquema proteccionista, con amplios beneficios para quienes invertían en el desarrollo industrial, engendró una industria poco competitiva y carente de calidad en sus productos, donde el Estado se erigió como el principal propietario. Esta industria, a veces empresarial y a veces partidista, no estaba capacitada para competir en el exterior.

El hallazgo de importantes reservas petroleras en el país supuso un alivio para el fatigado modelo por otros diez años, para finalmente entrar en una franca crisis que obligó a reorientar la economía hacia el mercado externo. En los gobiernos de Miguel de la Madrid (1982-1988), y especialmente de Carlos Salinas de Gortari (1988-1994), el patrón de desarrollo dio el viraje definitivo hacia una economía orientada al mercado externo, con la mirada dirigida particularmente a Estados Unidos, impulso que estuvo acompañado por una serie de transformaciones a nivel interno (Grayson, 2001).

Una economía lanzada al mercado externo; la virtual abolición de las barreras arancelarias; la firma del Tratado de Libre Comercio (TLCAN-NAFTA) con Estados Unidos y Canadá. Además, control de la emisión de circulante, abatimiento de la inflación, control del gasto corriente del gobierno y la venta de la mayoría de las empresas de éste, fueron decisiones que redefinie- 
ron el modelo económico y, de paso, el papel que tendría el Estado mexicano, que abandonó su papel de propietario por el de rector de la economía mexicana (ibidem).

\section{Un sistema electoral competitivo}

Estos cambios económicos fueron también portadores de profundos desplazamientos políticos, entre los cuales se puede mencionar una importante fisura y división en el interior del otrora compacto PRI, consecuencia -entre otros factores- de la reorientación económica, lo cual finalmente derivó en el surgimiento del PRD, especie de conciencia del ideario original de la Revolución mexicana, pero asimismo receptáculo de las inercias y vicios de siete décadas de burocracia y poder omnímodo.

El mercado abierto de productos y servicios implicó también el surgimiento de nuevos candidatos, con una imagen más atractiva para el México urbano que los tradicionales candidatos priistas de extracción popular y campesina. Los candidatos del PRI se empezaron a aparecer en franca desventaja en comparación a los más articulados y jóvenes candidatos de la oposición.

La mayor apertura hacia los mercados externos, especialmente el estadounidense, trajo consigo un mayor seguimiento de la prensa y actores internacionales de los acontecimientos políticos de México. En un contexto marcado por críticas emitidas por legisladores y medios estadounidenses, en el sentido de que el sistema político mexicano era "dictatorial, corrupto y unipartidista", se dio el reconocimiento oficial de los primeros triunfos del PAN en Baja California, Guanajuato y Chihuahua.

A partir de ese momento se apresuraron los avances en la consolidación de un sistema electoral más confiable y equitativo, haciéndose necesaria la creación del Instituto Federal Electoral, al que posteriormente se dio total autonomía, primer paso real para garantizar procesos electorales legítimos, confiables y transparentes. En muy poco tiempo la alternancia de partidos fue una realidad; las principales zonas metropolitanas del país empezaron a ser gobernadas por lo que en ese entonces de denominó oposición: Guadalajara, Mérida, Monterrey, Puebla y Tijuana, por mencionar algunas, bajo la férula del PAN; y el Distrito Federal regido por el PRD.

En unos cuantos años, el panorama político de México había sufrido un cambio dramático, pero además se abría la posibilidad de que el PRI perdiera la Presidencia de la República, pilar indiscutible del sistema político priista, situación que finalmente se dio en la elección del año 2000 con el triunfo de Vicente Fox. 


\section{La forma de comunicar del sistema priista}

Un estudio de Evelyn Stevens (1974) proporciona un análisis detallado de las prácticas de comunicación comunes en el sistema priista de década de 1960 . En éste podemos identificar cuatro aspectos relevantes a resaltar: a) respeto irrestricto a la figura presidencial; b) total dependencia de los medios con el gobierno y el consiguiente tratamiento positivo en las noticias y actividades del gobierno; c) prevalencia del rumor como una fuente fidedigna de información; $y, d)$ falta de credibilidad en los medios.

$\mathrm{Si}$ algo caracterizó al sistema priista fue la relevancia de la figura presidencial, que se reflejó muy fuertemente en el tratamiento que los medios le dieron a las actividades del Presidente de la República. En un análisis de la prensa de 1965 a 1966, Stevens no encontró ni una sola nota de crítica al Presidente, peculiaridad que llamó "glorificación de la figura presidencial". Como dato curioso destaca que en el plano interpersonal, por el contrario, la figura presidencial es constantemente ironizada y caricaturizada.

Los medios de comunicación dependían fuertemente, si no es que en forma total, de los recursos del Estado. En su análisis, Stevens reporta $30 \%$ de publicidad del gobierno entre los periódicos que revisó; esto y la publicidad indirecta (que no reportó, dado que aparece en forma de noticias) representan la fuente de ingresos más cuantiosa de los periódicos.

Los salarios de los periodistas frisan entre los 64 y los 400 dólares mensuales, pero el estilo de vida de muchos de ellos refleja entradas de 1,200 a 1,600 dólares. Resultó práctica común de los periodistas -reporteros de prensa, radio o televisión, indistintamente - recibir un apoyo económico en las distintas oficinas de gobierno que cubrían.

Otro rasgo importante del sistema de comunicación es la fuerte relevancia que se le dio al rumor, y que en muchos casos se mantiene. Es común entre las dependencias de gobierno contratar a una persona encargada de recolectar y difundir los principales rumores que circulan en el medio. Se considera la información de las personas tan relevante y vital "como un arma que se puede utilizar en contra de alguien" (p. 27).

Esta idea de la información como arma se veía reflejada en las encuestas, durante las cuales los entrevistados se resistían a contestar temas de carácter político ante el temor de enfrentar cualquier tipo de represalias.

Una expresión del peso del rumor es la "lectura entre líneas" de las columnas. Los mensajes de las columnas políticas son interpretados no por lo que expresan, sino por el significado soterrado que emiten, para lo cual es importante saber de quién es vocero el columnista y sobre qué aspecto hace referencia en particular.

Todas estas características daban como saldo una carencia de credibilidad en los medios de comunicación, e incluso en el sistema mismo. En este 
contexto, "comunicación" para los gobiernos era publicitar las obras y logros (reales y no reales) de sus gestiones, aparentando un sentido de avance a la sociedad y, en un segundo plano, de mensaje ideológico de los principios revolucionarios de justicia social e igualdad.

\section{Evaluación del gobierno, niveles socioeconómicos, intención de voto y filiación partidista}

Hay dos aspectos relevantes a considerar cuando se pretende analizar los factores que inciden o explican la evaluación ciudadana hacia los gobiernos en México: a) el contexto socioeconómico del país; $y, b$ ) la intención de voto y la filiación partidista de los ciudadanos.

\section{Contexto socioeconómico}

Una característica relevante de los electores en México son las altas expectativas que guardan respecto a sus gobiernos; esto es, esperan más de lo que realmente las instituciones gubernamentales les pueden dar, fenómeno explicado en parte por nuestra poca experiencia democrática, pero sobre todo por la desigualdad socioeconómica que caracteriza al país. ${ }^{3}$

Un rasgo a considerar de México - y de América Latina en general-es la gran desigualdad económica y social que existe entre su población (Garduño, 1998; Rodríguez, 1995; Székely, 1995; Valencia, et al., 2000). Mientras en Estados Unidos y Europa $20 \%$ de los habitantes más ricos recibe $42 \%$ del ingreso-consumo, en América Latina el $20 \%$ más rico es beneficiario de $62 \%$ (González, 2000).

Esta desigualdad afecta directamente la estructura socioeconómica de los países en América Latina. Por ejemplo, en México, de acuerdo a la Asociación Mexicana de Agencias de Investigación (AMAI), el grueso de la población se concentra en forma desproporcionada en el segmento más pobre: $60 \%$ de la población mexicana pertenece al nivel socioeconómico NSE " $D$ ". 4

3. Diversos autores señalan esta característica desde distintos ángulos. Para mayor detalle consultar Arredondo, (1996), García (1995) y Melucci (1999).

4. De acuerdo a la AMAI, los niveles socioeconómicos en México están conformados por tres grupos: a) el "A/B", que corresponde a $8 \%$ de la población, y que se conforma por lo general de personas con el mejor nivel de vida en el país. Acostumbran viajar al extranjero y por lo general son empresarios o ejecutivos de alto nivel, con estudios universitarios; b) el "C" (32\% de la población), conformado por las clases medias, profesionistas, residentes en zonas residenciales medianas y con acceso a tarjetas de créditos, entre otras características; y, c) el " $D$ " (60\% de la población), con grandes carencias materiales y educativas, 
Esta disparidad socioeconómica afecta la manera en que evalúa, si no la mayoría, sí una parte considerable de los mexicanos a sus gobiernos. Dentro de los ciudadanos del nivel NSE " $D$ " a menudo aflora la idea de que sus problemas se deben primordialmente a los gobiernos que nunca han ayudado a la población a salir adelante y, en consecuencia, su futuro depende principalmente de "la ayuda de los gobiernos".

La relación entre el nivel socioeconómico y la visión política ha sido reportada por algunos estudios. Almond y Sidney Verba (citado en McCann, 1996 y 1998) muestran cómo el interés en los asuntos políticos está significativamente determinado por el nivel socioeconómico; así, la diferencia entre el interés registrado en países del primer mundo y México está determinada por el segmento más desprotegido.

Dentro de la lógica de solución de sus necesidades, los habitantes que viven en condiciones de pobreza esperan que los gobernantes se comprometan a resolver problemas que rebasan las posibilidades de las funciones gubernamentales, trayendo como resultado un pronto desencanto, lo cual los induce a votar por un cambio de partido en la siguiente elección.

Este proceso presenta mucho más complejidades de las que se pueden describir en estas líneas, por lo que siempre habrá excepciones. También depende de la forma en que se desempeñan los gobiernos. Es cierto que en México nuestra clase política no es de las más competentes, y habrá otros casos en que la preferencia electoral no se manifieste de esta manera. Sin embargo, en términos generales y tomando como unidad de análisis a los electores del nivel NSE "D", sí se puede identificar esta característica.

Entre ciudadanos del nivel NSE "D" es común encontrar una lógica de evaluación de gobierno con una visión centrada en sus necesidades inmediatas. Desde su óptica, como el gobierno es el responsable de la situación de pobreza en que viven, la obligación de éste es garantizar en el corto plazo mejores empleos, salarios, servicios públicos y la erradicación de la pobreza en general.

Evaluación de gobierno, filiación partidista e intención de voto

Otro factor que incide en la evaluación de un gobierno es la filiación o preferencia partidista del ciudadano. Una persona que siente gran simpatía hacia un partido ("voto duro") generalmente evalúa a un gobierno del mismo partido político en términos más positivos; y por el contrario, a un gobierno de diferente partido con mucho más rigor y aspereza. Y como el desempeño

quienes generalmente se desarrollan en actividades manuales y de baja remuneración (Chiquiar, 1994). 
de un gobierno se conecta con la filiación partidista, bajo esta óptica se espera que el buen desempeño de un gobierno se conecte con el incremento en el número de sus simpatizantes partidistas. Sin embargo esta conexión no es nítida, y en el caso de México no se encontró trabajo alguno al respecto.

Lo mismo sucede con la relación entre evaluación de gobierno y patrones de voto, cuyo vínculo no se antoja claramente evidente, ni cómo se relaciona el desempeño de un gobierno con los resultados de una votación. Por ejemplo, se asume que el pésimo desempeño de un gobierno produce el voto de castigo que explica la derrota de un partido y la alternancia de diferentes institutos políticos en el poder. Sin embargo, cuando el gobierno está bien evaluado - por ejemplo el caso de Zedillo, con un rating de aprobación cercano a los 70 puntos- no siempre tiene como consecuencia una victoria del partido del gobernante, en este caso el PRI.

Es evidente la facilidad con la que podemos ligar el mal desempeño de un gobierno con su ulterior derrota electoral. Pero ya no es tan clara la conexión entre un buen desempeño en el gobierno y un posterior triunfo electoral. Es probable que las coyunturas electorales adquieran características mediáticas y temporales, de tal forma que la continuidad en muy pocas ocasiones es un tema de campaña. Esta cuestión se vuelve medular para el caso de la joven democracia mexicana. Flota aquí una pregunta: iqué caso tiene procurar hacer un buen gobierno, si finalmente el voto ciudadano probablemente no me va a favorecer?

Bajo esta perspectiva, se propone que todo gobierno sea evaluado a partir de tres variables:

1. Desempeño de gobierno: basado en un modelo de satisfacción de servicios, se propone construir un modelo de evaluación de gobierno en dos niveles de medición.

a) Global: qué tan satisfecho se siente el ciudadano con el desempeño de un gobierno en particular.

b) Por área: qué tan satisfecho se siente el ciudadano con el desempeño del gobierno en las distintas funciones tangibles e intangibles que el ciudadano le asigna.

2. Intención de voto: todo sistema de evaluación de gobierno tiene que reflejar la intención de voto, como consecuencia de la buena aplicación de una estrategia de gobierno y comunicación.

3. Filiación partidista: basado en el trabajo de Dillon (1982 y 1984) y González Molina (2000) sobre lealtad y switcheo, se propone una clasificación de los ciudadanos por su filiación partidista en cuatro segmentos:

a) Filiación leal o dura: se trata de aquellos ciudadanos que muestran una alta probabilidad de votar por un mismo partido.

b) Ciudadano volátil o switcher: a esta categoría pertenecen quienes muestran un patrón de votación a favor de dos o más partidos y una baja 
probabilidad de elegir siempre el mismo partido. Este segmento es el que pondera las diferentes propuestas y acciones de gobierno en términos de conveniencia; si lo benefician, entonces apoya la mejor propuesta.

c) Ciudadano antipartido: son quienes muestran una alta probabilidad de votar u opinar en contra de un partido y su gobierno; en consecuencia; votan para ver perder a un partido en particular.

d) Ciudadano abstencionista-oculto: tales electores muestran una alta propensión a la abstención u ocultan su verdadera opinión o intención de voto.

En términos de evaluación de gobierno, los switchers son el segmento más importante para una propuesta de gobierno o de comunicación. Satisfaciendo las necesidades de este segmento, se superan las expectativas de un ciudadano leal.

\section{Comunicación desde el gobierno}

En general se identifican dos perspectivas acerca de la comunicación en el gobierno: a) la perspectiva mercadológica, que comprende los enfoques de relaciones públicas, comunicación organizacional y comunicación corporativa; $y, b)$ la comunicación entendida como un proceso de desarrollo comunitario. A continuación se discutirán brevemente los acercamientos, con el objetivo de ubicar el presente trabajo dentro de una perspectiva que aborde idealmente los enfoques mercadológico y comunitario.

\section{Las perspectivas mercadotécnicas}

El área denominada "comunicación del sector público" es relativamente nueva. La consolidación del modelo de desarrollo capitalista como opción viable de desarrollo provocó, entre otras cosas, que los distintos gobiernos tuvieran la necesidad de planear su comunicación, con el fin de que los ciudadanos (clientes) percibieran valores agregados en las actuaciones de sus gobiernos (Graber, 1992).

La importancia de la comunicación radica en el hecho de que, dadas las condiciones de urbanización del mundo actual, gran parte de la acción de gobernar se da a través de los medios de comunicación y con los servicios a cargo del sector. De ahí que el ciudadano, aunque no conozca las obras y logros de los gobiernos, necesariamente se formará alguna idea de la actuación de sus gobiernos por esta vía.

A eso se debe que los gobiernos requieren de la planeación de sus estrategias de comunicación, con el fin de desdoblar un gobierno congruente 
con una serie de valores y misiones concretas que se proyectarán en cada una de las acciones de dicho gobierno.

A ciencia cierta, no existe un modelo de comunicación del sector público diseñado especialmente para el gobierno. Más bien, lo que se encuentra son propuestas desarrolladas en el sector privado, pero adaptadas al ámbito gubernamental. Estas aproximaciones se pueden clasificar en tres grandes grupos: comunicación organizacional, relaciones públicas y comunicación corporativa.

La perspectiva desde el enfoque de la comunicación organizacional busca identificar los flujos de información en los gobiernos y cómo se realizan las tomas de decisiones en las diferentes situaciones que se presentan. Sin embargo, este enfoque enfatiza sobremanera la estructura interna, de modo que sus receptores generalmente son los propios empleados.

Este enfoque carece de una visión más amplia, que permita planear y dirigirse a los públicos externos y a la opinión pública en general.

Un segundo enfoque comprende primordialmente las relaciones públicas, con un énfasis particular en la comunicación o envío de mensajes entre el sector público y los medios de comunicación, actores políticos y la ciudadanía en general, a través de campañas publicitarias.

Un tercer enfoque es la denominada comunicación corporativa, que ha tenido un vertiginoso desarrollo en la última década. Este enfoque contempla como primer paso identificar las diferentes audiencias a las cuales atiende el sector público. Una vez identificadas, es necesario realizar el diagnóstico de cada una de ellas. A partir de las necesidades identificadas, y en congruencia con los valores políticos y sociales del partido en el puesto, se diseña una estrategia pormenorizada para cada uno de sus públicos.

Este enfoque muestra la necesidad de construir una misión de gobierno, que debe ser plasmada en cada uno de los productos y programas de gobierno. Este enfoque ofrece la posibilidad de hacer uso de los otros dos, dado que se dirige a los distintos públicos o clientes del sector.

Desde nuestra perspectiva, consideramos este último enfoque como el ideal para una estrategia de comunicación desde el gobierno, con una limitación que responde particularmente a un contexto donde los ciudadanos comparten una serie de valores y necesidades similares. En el caso de México, como se vio anteriormente, este principio no se cumple a cabalidad, debido a que un importante sector de la población está más necesitado que otros, por lo cual es menester discutir algunos aspectos referentes al desarrollo comunitario. 
Como se discutió anteriormente, en México - como en América Latina en general- habita un gran sector de la población (60\%) que está más necesitado que otros de niveles socioeconómicos más fortalecidos. Este sector es el que explica en gran medida la constante aspiración de cambio entre los ciudadanos de México, y el cambio constante de partidos en los puestos de elección popular. $\mathrm{Y}$ es que al no verse cumplidas su expectativas (sobreestimadas) entonces votan por un cambio, repitiendo ese proceso una y otra vez.

Por esta razón, en el presente apartado se discute de un modo somero, pero no menos importante, un concepto de desarrollo comunitario que necesariamente se incluye como parte de nuestro concepto de comunicación en el sector público en México.

El paradigma dominante de desarrollo después de la Segunda Guerra mundial asumió que los países del tercer mundo podrían ser capaces de seguir el camino de los países desarrollados. Sin embargo, la experiencia no aconteció como se le quiso ver en ese entonces.

Muchos proyectos de desarrollo de esa época enfatizaron el peso de la cultura en el desarrollo. Desde esta óptica se dio un intenso debate de ideas entre quienes representaban esta corriente. Por ejemplo, la idea de la cultura de la pobreza de Lewis puede ubicarse desde este enfoque: los valores y creencias requieren ser cambiados para que el desarrollo económico tenga lugar (Mayo, 2000).

En este contexto, se le dio una fuerte relevancia a los medios de comunicación como instrumentos con un gran potencial para hacer posible el desarrollo económico en los países en vías de desarrollo. Típicamente, se diseñaban campañas de información basadas en la dramatización sobre problemas como el alcoholismo y la corrupción, por mencionar algunos, y se asumía que éstos tenían un gran impacto en las actitudes y conducta de las audiencias, en un paradigma típico de la bala mágica.

La principal crítica a esta visión es que sólo se centró en los aspectos culturales y en los factores individuales que inciden en el desarrollo comunitario, y pasó por alto los factores estructurales, como la mala distribución de la riqueza, un sistema educativo que garantice la igualdad de oportunidades, un sistema tributario que promueva la redistribución, la estabilidad económica, el manejo transparente de los recursos y las relaciones de poder-enriquecimiento de los caciques a nivel local, entre otros.

Promover el desarrollo comunitario necesariamente tiene que contemplar el nivel estructural que condiciona la permanencia de la pobreza, pero también los factores que operan a nivel de la cultura y a nivel individual.

En el nuevo contexto de gobiernos acotados por la globalización, la conformación de bloques económicos claramente definidos — particularmen- 
te en México-, la interdependencia con la economía estadounidense, la mala distribución de la riqueza, la pobreza y la necesidad de consolidar nuestro sistema democrático, se nos plantea la necesidad de implementar estrategias amplias que promuevan el desarrollo comunitario, lo mismo.en el ámbito urbano como en el rural; tanto con un sentido de manifestación de arte popular - expresado en los medios de comunicación-, como traducidos en la expresión de una política de desarrollo comunitario que involucre a la misma ciudadanía en la detección de sus necesidades y en la solución de las mismas.

\section{Rasgos metodológicos}

Los resultados que aquí se comentan representan la suma de dos estudios que se realizaron en dos diferentes zonas metropolitanas de México. A pesar de que se realizaron en periodos distintos, el diseño de la investigación fue el mismo en los dos casos.

El diseño de investigación se dividió en dos etapas: fase cualitativa y fase cuantitativa.

1. Fase cualitativa: se llevaron a cabo seis sesiones de grupo en cada una de las zonas metropolitanas, con las siguientes características:

- Objetivo de las sesiones: identificar las funciones y áreas del ayuntamiento desde la óptica del ciudadano.

- Unidad de análisis:

a) Dos sesiones con jóvenes entre 18 y 24 años de edad, hombres y mujeres, con credencial para votar con fotografía, switchers del partido del presidente municipal de la zona metropolitana.

b) Dos sesiones con amas de casa entre 25 y 34 años de edad, con credencial para votar con fotografía, switchers del partido del presidente municipal de la zona metropolitana.

c) Dos sesiones de grupo con empleados entre 25 y 34 años de edad, hombres y mujeres, con credencial para votar con fotografía, switchers del partido del presidente municipal de la zona metropolitana.

- Fecha de sesiones: en Guadalajara se realizaron las seis sesiones de grupo en junio de 2000; y en Puebla en febrero de 2001.

- Producto de las sesiones de grupo, se identificaron las funciones del gobierno, para incluirlas en un cuestionario para una medición cuantitativa. 
2. Fase cuantitativa: se realizó una medición cuantitativa a través de 600 entrevistas en cada una de las ciudades evaluadas, con las siguientes características:

- Objetivo de la medición cuantitativa: construir un modelo de gobierno de acuerdo a la satisfacción que manifiesta el ciudadano respecto a una medida global y una medida por función o área de gobierno.

- Unidad de análisis: personas de 18 años o más, residentes en las ciudades en estudio, con credencial para votar con fotografía.

- Margen de error: +/- 4\%, a un nivel de confianza de $95 \%$.

- Escala de medición: se empleó una escala Likert de 5 puntos con los siguientes valores: $1=$ Totalmente insatisfecho, $2=$ Algo insatisfecho, $3=\mathrm{Ni}$ satisfecho-ni insatisfecho, $4=$ Algo satisfecho y $5=$ Totalmente satisfecho.

- Técnica estadística para la generación del modelo de gobierno: General Linear Model.

- Fecha de campo: en Guadalajara se realizaron las 600 entrevistas en junio de 2000; y en Puebla en febrero de 2001.

- Producto de la encuesta: los resultados fueron diversos, dado que fueron estudios contratados por los ayuntamientos en cuestión. Aquí sólo se analizarán los que se refieren al modelo de gobierno y que responden a las tres preguntas de investigación planteadas en un inicio.

- Váriables. Se buscó construir un modelo de gobierno basado en dos tipos de variables:

a) Variable dependiente: satisfacción global, que se define como el grado en que el ciudadano se siente satisfecho con lo realizado en general por el gobierno municipal encabezado por su presidente. Esta variable se operacionalizó con un reactivo medido en escala Likert: ¿En general, qué tan satisfecho te encuentras con lo que ha realizado el actual Ayuntamiento de... (Guadalajara/Puebla), encabezado por... (Héctor Pérez Plazola/Mario Marín Torres), ¿dirías que te encuentras totalmente satisfecho, algo satisfecho, algo insatisfecho o totalmente insatisfecho?

b) Variables independientes: satisfacción por funciones o áreas, grado en que el ciudadano se siente satisfecho con lo realizado por el gobierno municipal en las funciones o áreas que son percibidas como responsabilidad del mismo. Estas variables se operacionalizaron con una serie de reactivos medidos en escala Likert, que variaron de acuerdo a las funciones que medían. 


\section{Evaluación global}

La evaluación global de los ciudadanos entrevistados en las dos zonas metropolitanas indica que se comportaron en forma similar; la diferencia de dos decimales no es estadísticamente significativa. El Ayuntamiento de Guadalajara, de filiación panista, reportó una media de 3.9, mientras que Puebla, de filiación priista, reportó 4.1 (véase gráfica 1).

\section{Gráfica 1}

Evaluación global de los ayuntamientos metropolitanos

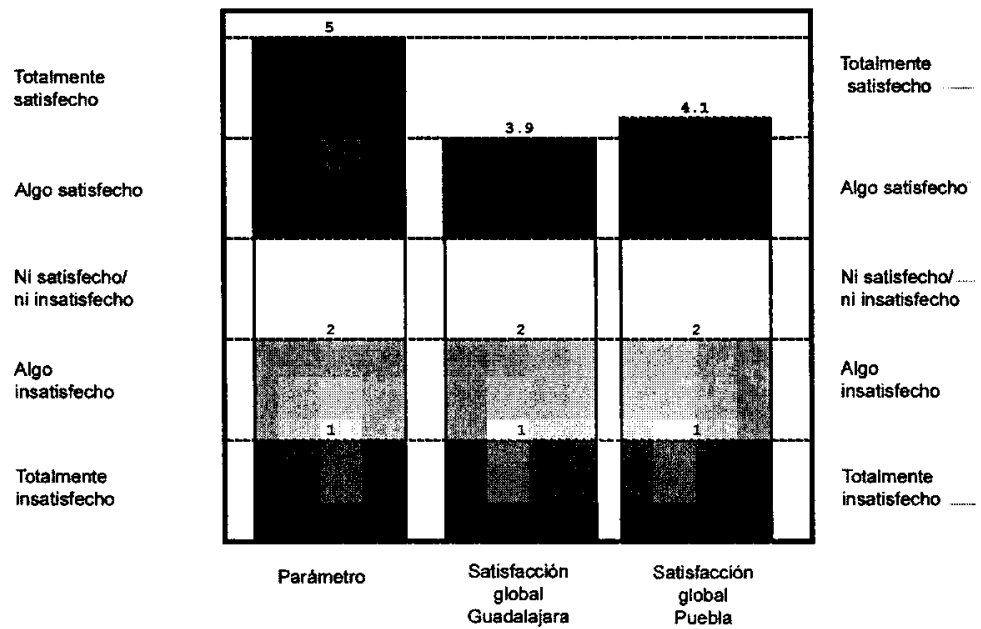

\section{Modelo de gobierno}

La diferencia más significativa no se encuentra en la satisfacción global del ciudadano, sino en el peso que le asignan los ciudadanos a las distintas funciones del gobierno municipal. Así vemos que los habitantes de la Guadalajara están satisfechos, en primer lugar, por los programas de participación ciudadana que ha emprendido el Ayuntamiento (predice $46 \%$ del desempeño global); en segundo lugar, por el servicio de agua potable y alcantarillado (29\%); en tercer lugar, por programas de atención al deporte $(15 \%)$ y; en cuarto lugar, por la eficiencia en los trámites en oficina (8\%; véase gráfica 2 ).

$\mathrm{La}$ intención de voto en este municipio favoreció ampliamente al PAN, el partido de origen del presidente municipal, Héctor Pérez Plazola, ya que 63\% de los ciudadanos entrevistados manifestaron su intención de votar por el PAN; mientras que $23 \%$ tuvo la intención de votar por el PRI. 
Gráfica 2

Modelo de gobierno de la ciudad de Guadalajara

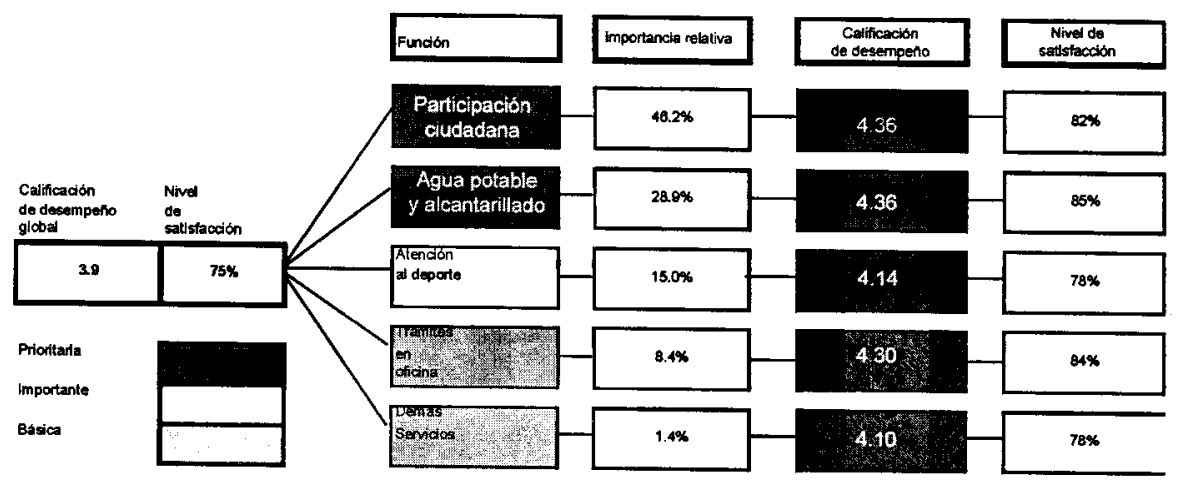

En cambio, en el caso del Ayuntamiento de Puebla, administración encabezada por Mario Marín Torres, perteneciente al PRI, los ciudadanos se sienten satisfechos, en primer lugar, por Parques y Jardines (predice $18 \%$ de la satisfacción global); en segundo lugar, pavimentación (16\%); en tercer lugar, seguridad pública (14\%); en cuarto lugar, mercados (14\%); en quinto lugar, alumbrado (13\%; véase gráfica 3$)$.

Contrariamente a lo encontrado en Guadalajara, la intención de voto no favoreció al partido del presidente municipal. El 38\% de los ciudadanos manifestaron su intención de votar por el PAN; mientras que $34 \%$ por el PRI.

Gráfica 3

Modelo de gobierno de la ciudad de Puebla

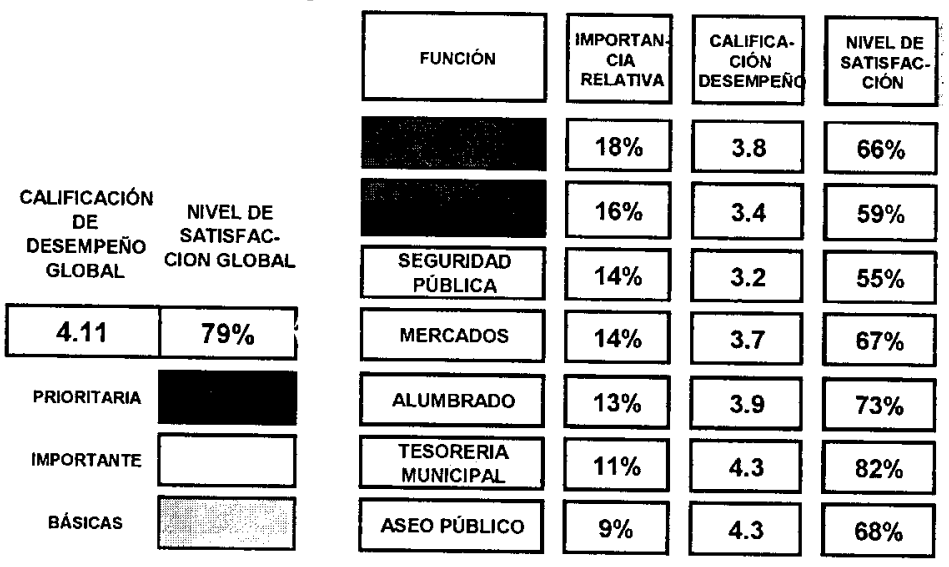


Con respecto a nuestras preguntas de investigación

1. ¿Cómo evalúan a sus gobiernos los ciudadanos mexicanos?

Los resultados aquí reportados sugieren dos niveles de evaluación: a) con respecto a la figura del presidente municipal; $y, b$ ) con respecto a una evaluación compleja.

a) El peso que tiene la figura del presidente municipal en la evaluación que hace el ciudadano con respecto al desempeño de un Ayuntamiento y su relación con la lealtad ciudadana, señalada por la intención de voto, indica que es importante pero no suficiente para que un partido permanezca en el poder.

Una evaluación positiva al desempeño de un gobernante no es suficiente para generar lealtad entre los ciudadanos. Por ejemplo, en el caso del Ayuntamiento de Puebla la calificación global de 4.1 alcanzada por el gobierno de Mario Marín es alto, los ciudadanos se encontraron entre algo y completamente satisfechos con su desempeño al frente del Ayuntamiento; sin embargo, no fue suficiente para generar lealtad entre los ciudadanos, dado que la intención de voto no favoreció a su partido en el momento en que se hizo la evaluación.

Mientras que en el caso del Ayuntamiento de Guadalajara, la calificación global de 3.9 reportada por el gobierno de Héctor Pérez Plazola lo ubica entre los gobiernos donde los ciudadanos se encuentran entre indiferentes y algo satisfechos con el desempeño del presidente municipal. Sin embargo, la intención de voto favorece ampliamente al partido del gobernante, en este caso el PAN.

Estos resultados, a primera vista parecen contradictorios, en el sentido de que se espera que un presidente municipal bien evaluado sea premiado con un voto a favor de su partido. A pesar de que diversos factores explican tal situación, como son: el contexto político muy particular de los municipios, las coyunturas, la evolución de la alternancia de partidos, la situación de los partidos a nivel nacional, estatal o local, entre otros, se puede afirmar que la imagen y una buena evaluación del presidente municipal no es suficiente para garantizar la continuidad de un partido en un Ayuntamiento.

b) La valoración más relevante es la que se relaciona con las diferentes funciones que tiene el gobierno desde la óptica del ciudadano. Por ejemplo, en el caso del Ayuntamiento de Guadalajara la calificación de las cinco áreas más importantes fue mayor a los 4 puntos, es decir los ciudadanos se encuentran entre algo y totalmente satisfechos. Mientras que en el caso de Puebla fue por debajo de ese nivel, excepto en las funciones de Tesorería Municipal y Aseo Público.

Estos resultados señalan que los ciudadanos tienen una visión amplia del gobierno en funciones, y que no sólo valoran la buena o mala imagen del gobernante, sino más bien los resultados en las distintas áreas o funciones 
asignadas como responsabilidad de la institución. Y dependiendo de la actuación del gobierno en estas áreas será la lealtad que tendrá el ciudadano con respecto al partido en el poder.

2. ¿Los ciudadanos perciben alguna diferencia entre gobiernos de distintos partidos?

A pesar de que las funciones de un Ayuntamiento están claramente definidas en el plano jurídico, y que los ciudadanos coinciden en gran medida con estas funciones, se presentan diferencias significativas en la forma en que los gobiernos de diferente filiación partidista están siendo valorados por sus ciudadanos.

La diferenciación se da en dos planos: competencia y la naturaleza de las funciones que predicen la satisfacción del ciudadano.

a) En el plano de la competencia es obvio que un gobierno que hace bien las cosas desde la óptica del ciudadano será bien evaluado por los mismos. Esto se puede ver muy claramente que en el caso del Ayuntamiento de Guadalajara, los ciudadanos valoraron que sus funciones eran realizadas en forma correcta, todas las áreas reportaron calificaciones mayores a los 4 puntos, entre algo y completamente satisfechos. Mientras que en el caso del gobierno municipal de Puebla los ciudadanos lo calificaron en niveles inferiores a los 4 puntos, entre indiferentes y algo satisfechos.

En este contexto, cuando los ciudadanos valoran que un gobierno cumple en forma satisfactoria con las diferentes funciones que le corresponde hacer, muy probablemente refrendarán su lealtad a ese partido. Así lo muestra el caso del Ayuntamiento de Guadalajara, que es calificado alto en cada una de sus funciones y, por consiguiente, registra una alta participación en la intención de voto el partido en el poder, es decir el PAN.

b) No obstante, la diferencia más significativa tiene lugar en las funciones que predicen la satisfacción del ciudadano. En el caso de los ciudadanos de Guadalajara, su satisfacción fue significativamente determinada por tres funciones; en primer lugar, participación ciudadana, con un peso de $46 \%$ para explicar la satisfacción global del Ayuntamiento; en segundo lugar, agua potable y alcantarillado, con $29 \%$ de peso; y en tercer lugar, atención al deporte, con un peso de $15 \%$.

Aquí el punto central es el peso que tiene participación ciudadana, que en sí misma no es una función tangible como lo es, por ejemplo, Pavimentación o Mercados. participación ciudadana es simultáneamente un instrumento de comunicación y un programa de gobierno. Es un instrumento de comunicación muy poderoso por tres razones: 
- Pone a consideración de la ciudadanía, en este caso los comités vecinales de las diferentes colonias de Guadalajara, una serie de programas de obra pública. Y desde ese momento se está comunicando la obra del gobierno y es conciliada con las necesidades del ciudadano al ponerse a su consideración.

- Promueve el consenso y la formación de ciudadanos responsables al involucrarlos en la resolución de los problemas que aquejan su realidad inmediata, en este caso su colonia.

- Legitima y brinda credibilidad a la obra del gobierno; al participar, los ciudadanos legitiman la obra que se llevará a cabo, y también les constan, por experiencia directa, las acciones y obras que emprende su gobierno.

Estos tres factores explican por qué el Ayuntamiento de Guadalajara generó lealtad entre sus ciudadanos y altas calificaciones en las diferentes funciones que son percibidas como de su incumbencia.

El hecho de que la participación ciudadana sea el principal predictor de la satisfacción ciudadana en Guadalajara redunda en un buena calificación en las demás áreas, dado que el ciudadano se involucró en la resolución de los problemas de su colonia, y en tal sentido las campañas de publicidad de obras de gobierno encuentran eco en la sociedad, pues a los ciudadanos les consta el trabajo que se realizó previo a las campañas.

En el caso de la ciudad de Puebla, los resultados indican que probablemente el gobierno de esta ciudad gastó recursos tanto como su similar de Guadalajara (es una hipótesis), pero que al concretar las obras y sus funciones de una manera tradicional -es decir que el gobierno planeó, diseñó y concretó las obras, sin crear un instrumento sistemático y formal de consulta ciudadana-, no logró el consenso y la credibilidad necesaria para conseguir la tan anhelada lealtad ciudadana (votación a su favor).

3. ¿Se puede pensar en un concepto de comunicación que sea algo más que promocionar la obra de gobierno, tan puesto en práctica en los actuales gobiernos municipales y estatales en el país?

No solamente es posible pensar en un modelo de comunicación que sea algo más que promocionar las obras del gobierno, o que busque vender una buena imagen del gobernante en turno o, en el peor de los casos, simular que los gobiernos están haciendo algo que definitivamente no están haciendo, sino que es necesario que los gobiernos en México busquen esquemas de comunicación amplios por tres razones:

a) La alternancia continua no es un beneficio por sí misma para la democracia en México, dado que promueve el desgaste y la credibilidad de todos los partidos políticos, y por ende del sistema en general, el ciudadano terminará con una percepción de que todos los partidos son lo mismo, que no 
importa la filiación de los gobernantes, al final todos desencantarán sus expectativas.

b) Muchas de las expectativas de los ciudadanos en México, principalmente en los sectores menos favorecidos, son una clara respuesta a sus carencias, que no son pocas. Desde esta óptica, los gobiernos son responsables de su situación de pobreza y, por ende, esperan que un gobierno los saque de esa condición. Por lo tanto, un esquema de comunicación tradicional resultará en desencanto, dado que las expectativas de los ciudadanos rebasan la capacidad real de los gobiernos.

c) Por lo tanto, es necesario involucrar a los ciudadanos en la resolución de los problemas que los afectan, dado que los gobiernos operan con recursos limitados, o al menos no suficientes para cubrir sus expectativas.

La participación ciudadana - que abarca desde la consulta a los ciudadanos en temas que afectan a todos hasta la administración de programas de gobierno- es una valiosa herramienta de comunicación en los ayuntamientos.

Programas de gobierno-comunicación pueden ser desarrollados a partir de la participación ciudadana, donde la idea principal que se transmite es que el ciudadano es el mismo gobierno (yo soy el gobierno). Desde este punto de vista, la comunicación supera al ya tradicional esquema de comunicación de promoción de obras (un gobierno cumplidor).

La participación ciudadana corresponde a un modelo de comunicación multisectorial, en la línea de los esquemas de desarrollo comunitario, con la diferencia significativa que tiene una aplicación urbana y no rural o semiurbana, como corresponde a los modelos de desarrollo comunitario.

La comunicación multisectorial tiene un doble objetivo: por un lado, promueve la formación de ciudadanos responsables al involucrarlos en la solución de sus propios problemas; y por otro lado, genera una cultura democrática entre la población, al establecer un diálogo en varios niveles, entre gobierno y ciudadanía, y entre grupos de ciudadanos, teniendo como resultado el consenso, como principal condición para que se lleve a cabo el cambio o los cambios que requiere el país en los distintos ámbitos: económico, social y político.

Finalmente, se plantea que los esquemas de gobierno municipal en México tendrán que orientar sus esfuerzos hacia áreas que generan valores agregados, que básicamente se concretan en tres aspectos fundamentales: a) participación ciudadana; b) calidad en el servicio; y, c) apoyo a grupos vulnerables. Estas tres áreas representan los ejes sobre los cuales los gobiernos podrán generar lealtad entre los ciudadanos en todo el país. 


\section{Bibliografía}

Arredondo, Vicente (1996), Hacia una nueva cultura ciudadana en México: elementos para transformar a la sociedad mexicana, México, DF, Universidad Iberoamericana.

Cochran, William G. (1980), Técnicas de muestreo, México, DF, Compañía Editorial Continental.

Chiquiar, Daniel (1994), "Estudio de niveles socioeconómicos", en MéxicoPrimera Versión, México, DF, Seminario de Actualización Profesional, AMAI.

Dillon, R. William y Madden, J. Thomas (1982), The Latent Structure Analysis, Chicago, American Marketing Association.

Dillon, R. William (1984), Multivariate Analysis: Methods and Applications, Nueva York, Wiley.

García Canclini, Néstor (1995), Consumidores y ciudadanos: conflictos multiculturales de la globalización, México, DF, Grijalbo.

Graber, Doris (1992), Public Sector Communication: How Organizations Manage Information, Washington, DC, Congressional Quarterly.

Grayson, George (2001), "Mexico Changing of the Guard", en Foreign Policy Association, Fall 2001, núm. 231, 78 pp.

Garduño Ríos, Sergio O. (1998), Indicadores de bienestar en México, 19401995, México, Venecia.

González Molina, Gabriel (2000), Cómo ganar las elecciones: estrategias de comunicación para candidatos y partidos, México, DF, Ediciones Cal y Arena.

Mayo, Marjorie (2000), Cultures, Communities, Identities, Great Britain, Antony Rowe.

Mc Cann, James (1996), "The Mexican Electorate in a North American Context: Assessing Patterns of Political Engagement", In Ai Camp, Roderic (1996), Polling for Democracy, Wilmington, Delaware, Scholarly Resources.

Mc Cann, James (1998), “The Changing Mexican Electorate: Political Interest, Expertise and Party Support in the 1980s and 1990s", en Serrano, Mónica (1998), Governing Mexico: Political Parties and Elections, Londres, University of London.

Melucci, Alberto (1999), Acción colectiva, vida cotidiana y democracia, México, DF, El Colegio de México. 
Rodríguez Hernández, Francisco (1995), "Crecimiento urbano y condiciones de vida en México: cambios en 1970-1990", en Comercio Exterior, octubre de 1995, vol. 45, núm. 10, pp.735-745.

Scheaffer, Richard L., William Mendenhall y Lyman Ott (1987), Elementos de muestreo, México, Grupo Editorial Iberoamérica.

Schatz, Sara (2000), Elites, Masses, and the Struggle for Democracy in México, Wesport, Connecticut, Praeger.

Stevens, Evelyn P (974), Protest and Response in Mexico, Cambridge, Massachusetts, The MIT Press.

Székely, Miguel (1995), "Poverty in Mexico During Adjustment”, en Review of Income and Wealth, septiembre de 1995, vol. 41, núm. 3, pp. 331-48.

Valencia Lomelí, Enrique, Mónica Gendreau y Ana María Tepichín Valle (2000), Los dilemas de la política social: ¿cómo combatir la pobreza?, Puebla, Universidad Iberoamericana. 